\title{
Association between telomere length and the risk of colorectal cancer: a meta- analysis of observational studies
}

\author{
Cho Naing ${ }^{1 *}$, Kyan Aung ${ }^{2}$, Pei Kuan Lai ${ }^{1}$ and Joon Wah Mak
}

\begin{abstract}
Background: Human chromosomes are capped and stabilized by telomeres. Telomere length regulates a 'cellular mitotic clock' that defines the number of cell divisions and hence, cellular life span. This study aimed to synthesize the evidence on the association between peripheral blood leucocytes (PBL) telomere length and the risk of colorectal cancer (CRC).

Methods: We searched relevant studies in electronic databases. When two or more observational studies reported the same outcome measures, we performed pooled analysis. All the analyses were performed on PBL using PCR. The odds ratio (OR) and its 95\% confidence interval $(\mathrm{Cl})$ were used to assess the strength of association.

Results: Seven studies (with 8 datasets) were included in this meta-analysis; 3 prospective studies, 3 retrospective studies and 1 study with a separate prospective and retrospective designs. The pooled analysis of 4 prospective studies (summary OR 1.01, 95\% Cl: 0.77-1.34, P:30\%) and 4 retrospective studies (summary OR 1.65, 95\% Cl: 0.96-2.83, $\left.P^{2}: 96 \%\right)$ showed no relationship between PBL telomere length and the CRC risk. A subgroup analysis of 2 prospective studies exclusively on females also showed no association between PBL telomere length and the CRC risk (summary OR, 1.17, 95\% Cl:0.72-1.91, P:57\%).

Conclusion: The current analysis is insufficient to provide evidence on the relationship between PBL telomere length and the risk of CRC. Findings suggest that there may be a complex relationship between PBL telomere length and the $C R C$ risk or discrepancy between genetics, age of patients and clinical studies. Future well powered, large prospective studies on the relationship between telomere length and the risk of $C R C$, and the investigations of the biologic mechanisms are recommended.
\end{abstract}

Keywords: Telomere, Colorectal cancer, Association, Meta-analysis

\section{Background}

Human chromosomes are capped and stabilized by telomeres, which not only protect them from damage but also have a role in regulating cellular senescence. After reaching a critical length, telomeres experience a double DNA change and cells will eventually enter (replication) senescence or cell death $[1,2]$, which may be due to a loss of chromosomal integrity [3]. Laboratory observations showed that telomere of human somatic cells act as a "mitotic clock", shortening with advancing age [1]. The

\footnotetext{
*Correspondence: cho3699@gmail.com

'School of Postgraduate Studies, International Medical University (IMU), Kuala Lumpur 57000, Malaysia

Full list of author information is available at the end of the article
}

exhaustion of proliferative potential of telomere, which is known as cellular senescence, occurred when telomeres cannot fulfil their normal protective functions [4].

There were an estimated 14.1 million cancer cases around the world in 2012. Of these, colorectal cancer (CRC) was the third most common cancer, accounting 1.36 million new case [5]. A considerable number of studies investigated the association between telomere length and human cancers including CRC. However, these individual studies reported inconsistently. Previous reviews in telomere sizes in cancer have reported CRC as a part [6-8], or were narrative reviews $[9,10]$. Human telomere length vary with age or cell types [11] and animal models have shown that this may have diverse effects in 
various organ systems [12]. Since the publication of these reviews, there has been a surge of published studies which assessed the association between telomere length and CRC. Thus, a meta-analysis addressing CRC and telomere length would be a useful addition to the current information in this area. Meta-analysis is a particular statistical strategy for bringing together the results of several studies (i.e. independent but 'comparable' studies) to produce a single estimate [13]. On the whole, the objective of the present study was to synthesize the evidence of published studies on the association between peripheral blood leucocytes (PBL) telomere length and the CRC risk.

\section{Methods}

The present study adhered to the preferred reporting items for systematic reviews and meta-analyses (PRISMA) statements [14].

\section{Study search}

We searched the relevant studies in the electronic databases such as PubMed, EMBASE, CANCERLIT, DARE (Database of Abstracts of Reviews of Effects), CINAHL, Web of Knowledge and Google Scholar. In initial searches, we used the broad search strategy 'telomere' OR 'telomere length' AND 'cancer' OR 'carcinoma' AND 'risk' OR 'epidemiology' OR 'pooled analysis' OR 'colon cancer' OR 'rectum cancer' OR 'colorectal cancer'. We modified the search strategy according to the requirements of different databases. Search was limited to publications in English through $7^{\text {th }}$ January 2016. We manually checked the reference lists of the relevant reviews and the included studies to find additional studies which could not be captured in electronic search.

\section{Study selection}

Studies were eligible if they (i) reported on associations between baseline telomere length and CRC (as defined by histology), (ii) included controls (in case-control studies) who were cancer-free or healthy person, (iii) selected participants (in cohort studies) with pre-existing CRC, (iv) selected samples from PBL, and (v) provided sufficient data to estimate the strength of association.

As described elsewhere [15] both prospective and retrospective designs were considered. In retrospective studies, telomere length was measured in DNA samples collected after diagnosis of CRC. In prospectively designed studies, this was done from participants prior to diagnosis or development of CRC. Retrospective studies included case-control and cross-sectional studies, while prospective studies included nested case-control and prospective cohort studies. Studies were excluded if they did not meet the inclusion criteria. For studies that enrolled overlapping participants, only the studies with larger samples were included.

\section{Data extraction}

Two authors independently screened the titles and abstracts, and assessed the full text, if deemed relevant for this review. Any discrepancy was resolved by discussion. The selected articles were reviewed to determine their eligibility for the current review. The data from the included studies were extracted using a piloted data extraction sheet. We extracted the following information from each of the included studies: author, year of publication, year of study, country, study design, type of CRC (i.e. colon cancer, rectal cancer, CRC), sample type, sample source, assay and outcomes reported. Any disagreement in data extraction was resolved by consensus or involving a senior author.

\section{Methodological assessments}

One author appraised the methodological quality of each included studies using Newcastle-Ottawa Scale (NOS) [16]. This was cross-checked by another author. The study quality rating of each study was judged based on the participants' selection (four stars), the comparability of the study groups (two stars) and the assessment of exposure (three stars). The highest total score for a study was nine. Any discrepancy between the two assessors was resolved by consensus and by taking advice from a senior author.

\section{Statistical analysis}

We extracted study specific OR and corresponding 95\% confidence interval (CI) from the included studies (i.e. for the association between shortest Q4 vs longest Q1, Q1 vs Q4 or below vs above the median length). We assumed that the relative risk (RR) from cohort studies approximates the odds ratio (OR) from case-control studies [17]. We transformed OR and its 95\% CI into natural log OR and its standard error (SE) of log OR before meta-analysis [18]. Statistical heterogeneity between studies was assessed with the $I^{2}$ test, which indicates the variation in effect estimates due to (true) heterogeneity rather than withinstudy error [19]. A value of $\geq 50 \%$ indicates substantial heterogeneity [18, 19]. A random-effects model with inverse variance weighting was used to calculate the pooled OR and its 95\% CI as there was substantial heterogeneity between studies [20]. Otherwise, we used a fixed-effect model.

All statistical analyses were performed with RevMan 5.3 [21]. Dataset is freely available from the corresponding author $(\mathrm{CN})$ for non-commercial purpose.

\section{Results}

\section{Study characteristics}

The study selection process is provided in a flowchart (Additional file 1: Figure S1). After screening titles and 
abstracts, 20 full texts were retrieved; seven studies (with 8 data sets) [22-28] were selected for inclusion in this meta-analysis. Thirteen studies [29-41] were excluded as they did not meet the inclusion criteria. Reasons for the exclusion were summarized in Additional file 2: Table S1.

Table 1 presents the characteristics of the included studies. Three studies were prospectively designed studies [22, 23, 25], three studies were retrospective studies [26-28], and one study was done with prospective and retrospective designs, separately [24]. Two studies assessed exclusively with female participants [23, 25], while one study with males [22]. These studies were published between 2009 and 2014. Age of participants ranged from 18 to 82 years.

Two studies each were done in China $[25,28]$ and the UK $[24,25]$ The remaining three studies were from the United States [22, 23, 27]. Three studies [22, 23, 27] have provided separate components in CRC, indicating most were colon cancer $(70.3-78.8 \%)$ and the rest were rectal cancer (21.2-29.7\%). In all studies, DNA sources was extracted from PBL and used qPCR to measure the telomere length. The Pellatt study used immortalized cell lines for colon cancer and PBL for rectal cancer [26]. Methodological quality of the included studies was provided (Additional file 3: Table S2). All studies were high in methodological quality, achieving 7-9 stars [22-28].

\section{Summary estimates}

Overall, there is no significant relationship between telomere length measured in PBL DNA and the CRC risk in both retrospective and prospective studies. The summary OR in 4 retrospective studies was 1.65 (95\% CI: 0.96-2.83) with substantial statistical heterogeneity $\left(I^{2}: 96 \%\right)$. The summary OR $(1.01,95 \% \mathrm{CI}: 0.77-1.34)$ in four prospective studies was also with significant heterogeneity $\left(I^{2}: 30 \%\right)$ (Fig. 1).

\section{Subgroup analysis}

When we analyzed two prospective studies solely on female participants $[23,25]$, there also was no significant association between telomere length and the CRC risk (summary OR, 1.17; 95\% CI:0.72-1.91, $I^{2}: 57 \%$ ) (Fig. 2). Due to a paucity of data, we were not able to perform subgroup analysis with age groups.

\section{Discussion}

Based on the available data, the present study has provided insights into the relationship between PBL telomere length and the CRC risk. Due to small number of studies the results from the current analysis is insufficient to provide evidence on the role of PBL telomere length and CRC risk.

\section{Telomere length in CRC}

Findings in this review showed there is no significant relationship between telomere length measured in $\mathrm{PBL}$ DNA and the CRC risk in both retrospective and prospective studies. There are many possible reasons for such relationship. The risk of CRC might be elevated by shorter or longer length of telomere, indicating a Ushape association [25]. It is possible that loss of telomeric DNA in relation to degradation or incomplete replication is apparently balanced by telomere elongation $[1,42,43]$. This also implied that telomere length in an 'appropriate range' may be necessary to maintain chromosomal stability and normal programmed cell death - functions which are protective against tumour development $[25,44]$. It has been hypothesized that cells with long telomeres may favour a delayed cell senescence and apoptosis, leading to an increased chance of various genetic and environmental insults and subsequent accumulations of genetic abnormalities attributed to a higher risk of carcinogenic transformation [25].

On the other hand, the relationship between short telomere length and the CRC risk is biologically plausible [28]. A large proportion of human cancers are made up of cells with very short telomeres $(5 \mathrm{~kb})$, which is attributable to telomere dysfunction [45]. Although the exact mechanism is not fully understood, it is possible that, in some situations, cells with critically short telomere length may reactivate the telomerase enzyme, and this further promotes malignant transformation [28]. The putative pathway for telomere-associated neoplasia is that shortening of telomeres in the colorectal mucosa increases the chromosomal susceptibility to instability [40] as well as the microsatellite instability [47].

Reasons for no significant associations in our findings also rest on discrepancy of the study participants such as gender, age, life styles and the study design related factors (e.g. sample size, prospective or retrospective study). For instance, an association of telomere length and the CRC development in age-depending manner was shown in an individual study included in this analysis [28]. Older patients with shorter telomeres may be prone to an increased risk for various types of cancers due to telomere crisis [28] and subsequent chromosomal aberrations [25] and the diseases that result [25]. Extremely long telomere in younger individuals may be indicative of dysregulation in telomere regulation process [28].

We found a change in the effect estimates in a subgroup analysis solely with females. This highlighted that hormonal influence (estrogen) on the length of telomere needed to be given due attention in interpreting the results. Studies documented that the rate of terminal restriction fragment length shortening per year in men was significantly greater than that in women [45]. An estrogen-responsive element is present in telomerase 


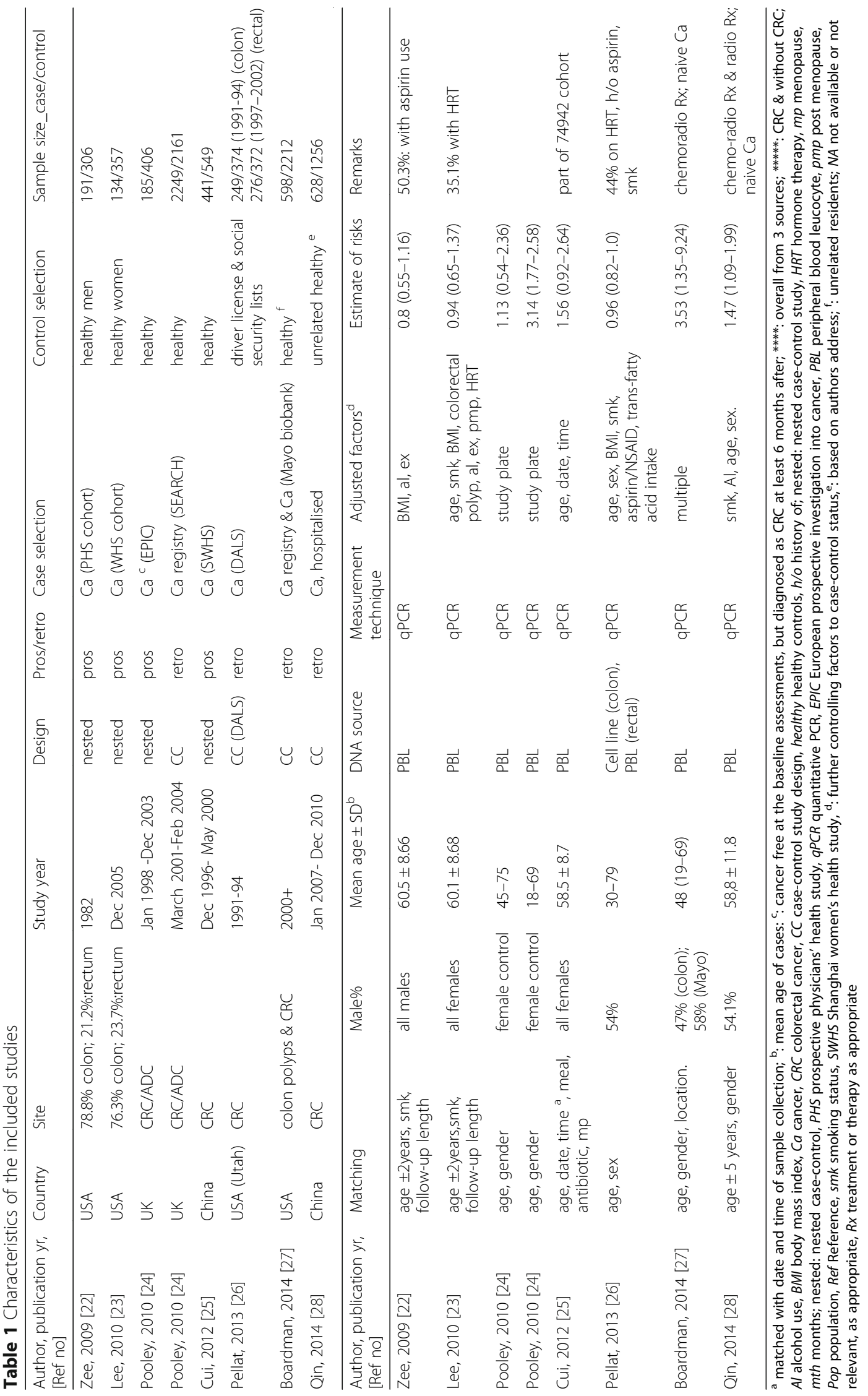




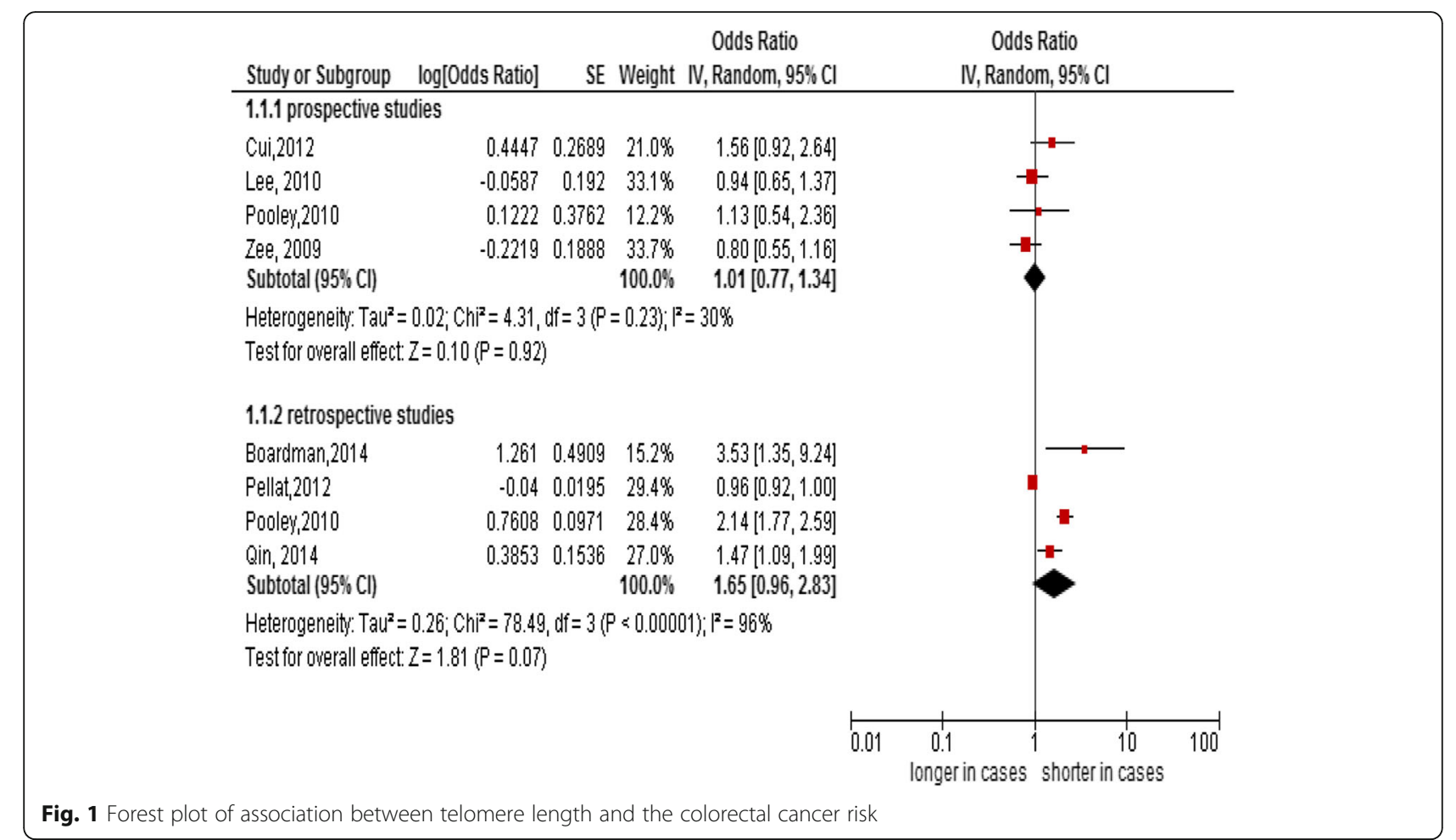

reverse transcriptase (hTERT), so the hormone can stimulate telomerase [46]. This might have reduced the shortening of telomere. Another possible mechanism is in the light of reactive oxygen species (ROS), which are number of reactive molecules and free radicals derived from molecular oxygen. As such, hydrogen peroxide and other ROS accelerate telomere erosion at least in cultured cells. Women are known to produce fewer reactive oxygen species [42] and this might contribute to less telomere attrition.
Most of the primary studies in this review have estimated an individual's telomere length from a single blood collection. In a study, multiple measurements showed a good reliability (intraclass correlation coefficient 0.64) indicating a one-time measurement is a good representation of an individual's telomere length within a short period of time [48]. Hence, a one-time determination in the primary studies would not affect the confidence in the effect estimate of the current review.

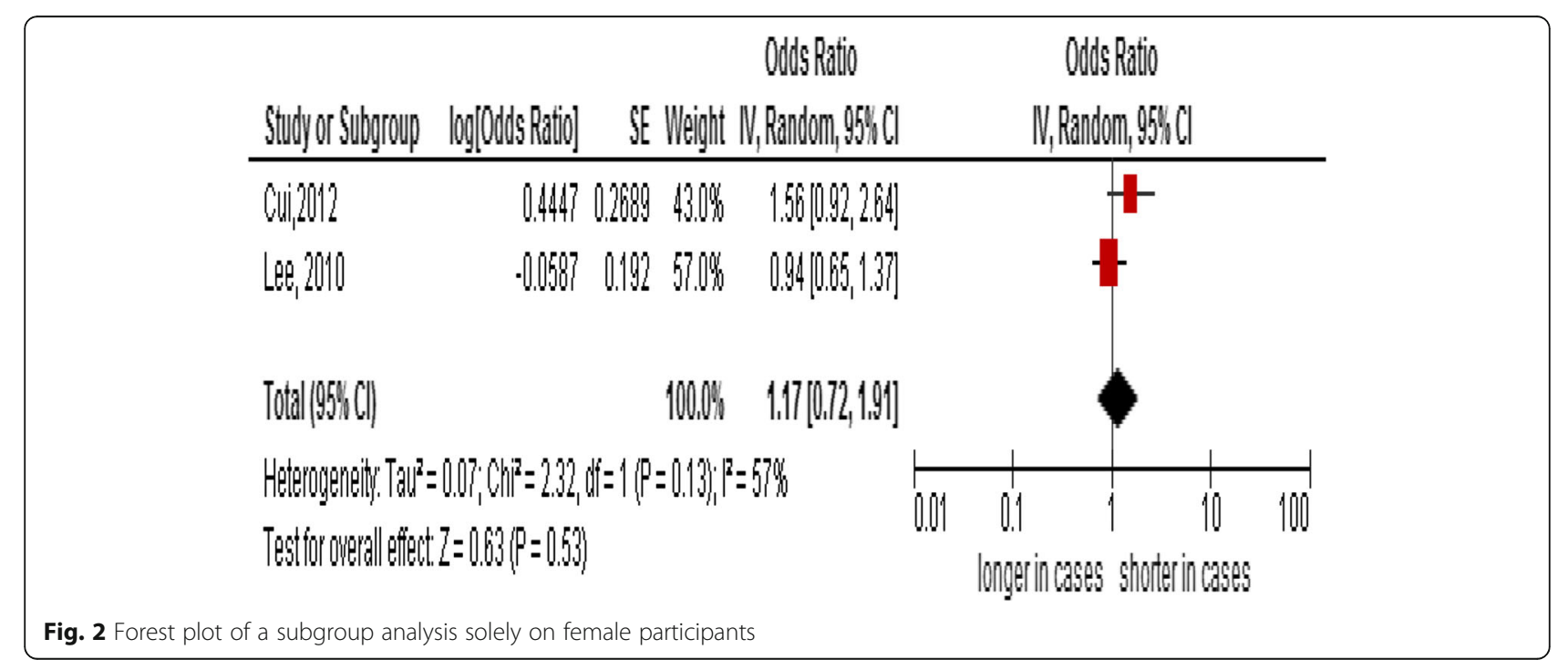




\section{Study limitations and strengths}

There are some limitations that need to be acknowledged. Although we had done an exhaustive literature search, it was possible that some publications might have been overlooked, especially when abstracts in English were not available. Variation in sample collection time in relation to study design among included studies is a concern. Samples in the prospective study were collected (many) years before diagnosis and therefore changes in participant's diet and lifestyle related factors and drug history (e.g. treatment with estrogen in women) overtime could have influenced telomere length, or conversely telomere length could be influenced by diagnosis and treatment in the retrospectively designed study [24, 27].

Variation in measurement methods of telomere lengths in the primary studies could have affected the effect estimates. This was, however, not the case in the present review as all studies applied qPCR for the measurement of telomere length. Stage-specific relationship could not be analysed as staging of disease with Dukes' or TNM systems had been provided inconsistently in a few studies. This might lead to a misclassification bias in interpreting the results.

Since relevant data were limited, we could not adjust for confounding factors. For instance, age of patients was likely to be a confounding factor for the association between PBL telomere length and the CRC risk. It is difficult to render the pooled analysis stratified by age groups, which were not consistently classified among these studies. Individual studies $[45,49]$ as well as a systematic review had suggested an interaction between aspirin or non-steroidal anti-inflammatory drug use $[49,50]$ and genes encoding transcription factor [49] or a combined effect of life style with gene encoding [27] in oncogenesis, inflammatory and drug metabolic pathways in relation to risk of CRC [50]. Hence, our results might be influenced by these confounding factors.

Due to a small number of studies with limited sample sizes, there is a limited power to detect a true difference of telomere length between cases and controls. It calls for future well designed, large prospective studies in this field.

\section{Conclusion}

The current analysis is insufficient to provide evidence on the relationship between PBL telomere length and the risk of CRC. Findings suggest that there may be a complex relationship between PBL telomere length and CRC risk, suggesting a U-shaped relationship or discrepancy between genetics, age of patients and clinical studies. Future well powered, large prospective studies on the relationship between PBL telomere length and the risk of CRC, and the investigations of the biologic mechanisms are recommended.

\section{Additional files}

Additional file 1: Figure S1. Study selection process. (DOC 29 kb)

Additional file 2: Table S1. Excluded studies and reason for exclusion. (DOC $26 \mathrm{~kb}$ )

Additional file 3: Table S2. The methodological quality of the included studies. (DOC $41 \mathrm{~kb}$ )

\section{Abbreviations}

Cl: Confidence interval; CRC: Colorectal cancer; OR: Odds ratio; PBL: Peripheral blood leucocytes; ROS: Reactive oxygen species; SE: Standard error

\section{Acknowledgements}

The authors thank the participants and researchers of the primary studies in this meta-analysis and the International Medical University (IMU), Malaysia for allowing us to perform this study. We wish to thank the anonymous reviewers and the editors for giving the comments and valuable inputs to improve the manuscript.

Funding

None.

Availability of data and material

Data supporting the results were reported in the manuscript.

\section{Authors' contribution}

JWM: conceptualized and coordinated the study; JWM, KA, CN: designed; KA, CN, LPK: extracted data, assessed quality of studies; CN: analyzed; JWM, KA, CN: interpreted; CN: revised the manuscript; JWM, KA, LPK: contributed additional information. All authors approved the final version of the revised manuscript.

\section{Competing interests}

The authors declare that they have no competing interests.

\section{Consent for publication}

Not applicable.

\section{Ethics approval and consent to participate}

Ethics approval and consent were waived by the Joint Research and Ethics Committee (JC) at the International Medical University in Kula Lumpur of Malaysia as this study was exclusively performed with the published data.

\section{Author details}

${ }^{1}$ School of Postgraduate Studies, International Medical University (IMU), Kuala Lumpur 57000, Malaysia. ${ }^{2}$ School of Medicine, International Medical

University (IMU), Kuala Lumpur, Malaysia.

Received: 30 January 2016 Accepted: 13 December 2016

Published online: 05 January 2017

References

1. Harley CB. Telomere loss: mitotic clock or genetic time bomb? Mutat Res. 1991;256:271-82.

2. O'Sullivan RJ, Karlseder J. Telomeres: protecting chromosomes against genome instability. Nat Rev Mol Cell Biol. 2010;11:171-81.

3. Slagboom PE, Droog S, Boomsma DI. Genetic determination of telomere size in humans: a twin study of three age groups. Am J Hum Genet. 1994;55:876-82.

4. D'Adda di Fagagna F, Reaper PM, Clay-Farrace L, Fiegler H, Carr P, von Zglinicki T, et al. A DNA damage checkpoint response in telomereinitiated senescence. Nature. 2003;426:194-8.

5. GLOBOCAN. Cancer Incidence and Mortality Worldwide: IARC Cancer Base No. 11 [Internet]. 2012:: http://globocan.iarc.fr/Default.aspx. Last accessed 3 Jan 2016.

6. Ma H, Zhou Z, Wei S, Liu Z, Pooley KA, Dunning AM, et al. Shortened telomere length is associated with increased risk of cancer: a meta-analysis. PLoS One. 2011;6:e20466. 
7. Wentzensen IM, Mirabello L, Pfeiffer RM, Savage SA. The association of telomere length and cancer: a meta-analysis. Cancer Epidemiol Biomarkers Prev. 2011;20:1238-50.

8. Prescott J, Wentzensen IM, Savage SA, De Vivo I. Epidemiologic evidence for a role of telomere dysfunction in cancer etiology. Mutat Res. 2012;730:75-84.

9. Gancarcikova M, Zemanova Z, Brezinova J, Berkova A, Včelíkova S, Smigova J, et al. The role of telomeres and telomerase complex in haematological neoplasia: the length of telomeres as a marker of carcinogenesis and prognosis of disease. Prague Med Rep. 2010;111:91-105.

10. Lim KG. A review of colorectal cancer research in Malaysia. Med J Malaysia. 2014;69(Suppl A):23-32.

11. Granger MP, Wright WE, Shay JW. Telomerase in cancer and aging. Crit Rev Oncol Hematol. 2002:41:29-40.

12. Lee HW, Blasco MA, Gottlieb GJ, Horner JW, Greider CW, DePinho RA, et al. Essential role of mouse telomerase in highly proliferative organs. Nature. 1998;392:569-74.

13. Sackett DL, Rosenberg WM, Gray JA, Haynes RB, Richardson WS. Evidence based medicine: what it is and what it isn't. Clin Orthop Relat Res. 2007;455:3-5.

14. Moher D, Liberati A, Tetzlaff J, Altman DG, PRISMA Group. Preferred reporting items for systematic reviews and meta-analyses: the PRISMA statement. Ann Intern Med. 2009;51:264-9.

15. Haycock PC, Heydon EE, Kaptoge S, Butterworth AS, Thompson A, Willeit P. Leucocyte telomere length and risk of cardiovascular disease: systematic review and meta-analysis. BMJ. 2014;349:94227.

16. Wells GA, Shea B, O'Connell D, Peterson J, Welch V, Losos M, et al. The Newcastle-Ottawa Scale (NOS) for assessing the quality of non-randomised studies in meta-analyses. Ottawa Hospital Research Institute: Ottawa; 2011. http://www.ohri.ca/programs/clinical_epidemiology/oxford.asp. Last accessed 3 Jan 2016.

17. Greenland S, Thomas DC, Morgenstern H. The rare-disease assumption revisited. A critique of estimators of relative risk for case-control studies. Am J Epidemiol. 1986;124:869-83.

18. Higgins JPT, Green S, eds. Cochrane Handbook for Systematic Reviews of Interventions.Version 5.1.0 (updated March 2011). The Cochrane Collaboration; 2011. Available at: http://training.cochrane.org/handbook. Last accessed 3 Jan 2016

19. Higgins JPT, Thompson SG, Deeks JJ, Altman DG. Measuring inconsistency in meta-analyses. BMJ. 2003;327:557-60.

20. DerSimonian R, Laird N. Meta-analysis in clinical trials. Control Clin Trials. 1986;7:177-88

21. Review Manager (RevMan) [Computer program]. Version [5.3]. Copenhagen: The Nordic Cochrane Centre, The Cochrane Collaboration, 2014. http:// www.cochrane.org/about-us/citing-our-products.

22. Zee RY, Castonguay AJ, Barton NS, Buring JE. Mean telomere length and risk of incident colorectal carcinoma: a prospective, nested case-control approach. Cancer Epidemiol Biomarkers Prev. 2009;18:2280-2.

23. Lee IM, Lin J, Castonguay AJ, Barton NS, Buring JE, Zee RY. Mean leukocyte telomere length and risk of incident colorectal carcinoma in women: a prospective, nested case-control study. Clin Chem Lab Med. 2010;48:259-62.

24. Pooley KA, Sandhu MS, Tyrer J, Shah M, Driver KE, Luben RN, et al. Telomere length in prospective and retrospective cancer case-control studies. Cancer Res. 2010;70:3170-6.

25. Cui Y, Cai Q, Qu S, et al. Association of leukocyte telomere length with colorectal cancer risk: nested case-control findings from the Shanghai Women's Health Study. Cancer Epidemiol Biomarkers Prev. 2012;21:1807-13.

26. Pellatt AJ, Wolff RK, Lundgreen A, Cawthon R, Slattery ML, et al. Genetic and lifestyle influence on telomere length and subsequent risk of colon cancer in a case control study. Int J Mol Epidemiol Genet. 2012;3:184-94.

27. Boardman LA, Litzelman K, Seo S, Johnson RA, Vanderboom RJ, Kimmel GW et al. The association of telomere length with colorectal cancer differs by the age of cancer onset. Clin Transl Gastroenterol. 2014;5:e52.

28. Qin Q, Sun J, Yin J, Liu L, Chen J, Zhang Y, et al. Telomere length in peripheral blood leukocytes is associated with risk of colorectal cancer in Chinese population. PLoS One. 2014:9:e88135.

29. Engelhardt M, Drullinsky P, Guillem J, Moore MA. Telomerase and telomere length in the development and progression of premalignant lesions to colorectal cancer. Clin Cancer Res. 1997:3:1931-41.

30. Engelhardt M, Albanell J, Drullinsky P, Han W, Guillem J, Scher HI, et al. Relative contribution of normal and neoplastic cells determines telomerase activity and telomere length in primary cancers of the prostate, colon, and sarcoma. Clin Cancer Res. 1997;3:1849-57.

31. Takagi S, Kinouchi Y, Hiwatashi N, Chida M, Nagashima F, Takahashi S, et al. Telomere shortening and the clinicopathologic characteristics of human colorectal carcinomas. Cancer. 1999;86:1431-6.

32. Takagi S, Kinouchi Y, Hiwatashi N, Nagashima F, Chida M, Takahashi S, et al. Relationship between microsatellite instability and telomere shortening in colorectal cancer. Dis Colon Rectum. 2000;43(Suppl):S12-7.

33. Gertler R, Rosenberg R, Stricker D, Werner M, Lassmann S, Ulm K, et al. Prognostic potential of the telomerase subunit human telomerase reverse transcriptase in tumor tissue and nontumorous mucosa from patients with colorectal carcinoma. Cancer. 2002;95:2103-11.

34. Gertler R, Rosenberg R, Stricker D, Friederichs J, Hoos A, Werner M, et al. Telomere length and human telomerase reverse transcriptase expression as markers for progression and prognosis of colorectal carcinoma. J Clin Oncol. 2004;22:1807-14.

35. Garcia-Aranda C, de Juan C, Diaz-Lopez A, Sanchez-Pernaute A, Torres AJ, Diaz-Rubio E, et al. Correlations of telomere length, telomerase activity and telomeric-repeat binding factor 1 expression in colorectal carcinoma. Cancer. 2006;106:541-51.

36. O'Sullivan J, Risques RA, Mandelson MT, Chen L, Brentnall TA, Bronner MP, et al. Telomere length in the colon declines with age: a relation to colorectal cancer? Cancer Epidemiol Biomarkers Prev. 2006;15:573-7.

37. Bautista CV, Felis CP, Espinet JMR, Garcia JB, Salas JV. Telomerase activity and telomere length in the colorectal polyp-carcinoma sequence. Rev Esp Enferm Dig. 2009;101:179-86.

38. Jones AM, Beggs AD, Carvajal-Carmona L, Farrington S, Tenesa A, Walker M, et al. TERC polymorphisms are associated both with susceptibility to colorectal cancer and with longer telomeres. Gut. 2012;61:248-54.

39. Riegert-Johnson DL, Boardman LA, Crook JE, Thomas CS, Johnson RA, Roberts ME, et al. Shorter peripheral blood telomeres are a potential biomarker for patients with advanced colorectal adenomas. Int J Biol Markers. 2012;27:e375-80.

40. Chen Y, Qu F, He X, Bao G, Liu X, Wan S, Xing J. Short leukocyte telomere length predicts poor prognosis and indicates altered immune functions in colorectal cancer patients. Ann Oncol. 2014;25:869-76.

41. Seguí N, Guinó E, Pineda M, Navarro M, Bellido F, Lazaro C, et al. Longer telomeres are Associated with cancer risk in MMR-proficient hereditary nonpolyposis colorectal cancer. PLoS One. 2014;9(2):e86063.

42. Bodnar AG, Ouellette M, Frolkis M, Holt SE, Chiu CP, Morin GB, et al. Extension of life-span by introduction of telomerase into normal human cells. Science. 1998:279:349-52

43. Ehrlenbach S, Willeit P, Kiechl S, Willeit J, Reindl M, Schanda K, et al. Influences on the reduction of relative telomere length over 10 years in the population-based Bruneck study: introduction of a well-controlled highthroughput assay. Int J Epidemiol. 2009;38:1725-34.

44. Hou L, Zhang X, Gawron AJ, Liu J. Surrogate tissue telomere length and cancer risk: shorter or longer? Cancer Lett. 2012:319:130-5.

45. Nawrot TS. The significance of telomerase activation and cellular immortalization in human cancer. Mutagenesis. 2002;17:539-50.

46. Kyo S(1), Takakura M, Kanaya T, Zhuo W, Fujimoto K, Nishio Y, Orimo A, Inoue M. Estrogen activates telomerase. Cancer Res. 1999:59:5917-21.

47. Rampazzo E, Bertorelle R, Serra L, Terrin L, Candiotto C, Pucciarelli S, et al. Relationship between telomere shortening, genetic instability, and site of tumour origin in colorectal cancers. Br J Cancer. 2010;102:1300-5.

48. Kim HR, Kim YJ, Kim HJ, Kim SK, Lee JH. Telomere length changes in colorectal cancers and polyps. J Korean Med Sci. 2002;17:360-5.

49. Pellatt AJ, Wolff RK, Herrick J, Lundgreen A, Slattery ML. TERT's role in colorectal carcinogenesis. Mol Carcinog. 2013:52:507-13.

50. Andersen V, Vogel U. Systematic review: interactions between aspirin, and other nonsteroidal anti-inflammatory drugs, and polymorphisms in relation to colorectal cancer. Aliment Pharmacol Ther. 2014;40:147-59. 